\title{
Approach and Development of Effective Models for Simulation of Thermal Stratification and Mixing Induced by Steam Injection into a Large Pool of Water
}

\author{
Hua Li, Walter Villanueva, and Pavel Kudinov \\ Division of Nuclear Power Safety, Royal Institute of Technology (KTH), Roslagstullsbacken 21, 10691 Stockholm, Sweden \\ Correspondence should be addressed to Pavel Kudinov; pavel@safety.sci.kth.se
}

Received 10 January 2014; Accepted 22 May 2014; Published 17 July 2014

Academic Editor: Leon Cizelj

Copyright (c) $2014 \mathrm{Hua} \mathrm{Li}$ et al. This is an open access article distributed under the Creative Commons Attribution License, which permits unrestricted use, distribution, and reproduction in any medium, provided the original work is properly cited.

\begin{abstract}
Steam venting and condensation in a large pool of water can lead to either thermal stratification or thermal mixing. In a pressure suppression pool (PSP) of a boiling water reactor (BWR), consistent thermal mixing maximizes the capacity of the pool while the development of thermal stratification can reduce the steam condensation capacity of the pool which in turn can lead to pressure increase in the containment and thereafter the consequences can be severe. Advanced modeling and simulation of direct contact condensation in large systems remain a challenge as evident in commercial and research codes mainly due to small time-steps necessary to resolve contact condensation in long transients. In this work, effective models, namely, the effective heat source (EHS) and effective momentum source (EMS) models, are proposed to model and simulate thermal stratification and mixing during a steam injection into a large pool of water. Specifically, the EHS/EMS models are developed for steam injection through a single vertical pipe submerged in a pool under two condensation regimes: complete condensation inside the pipe and chugging. These models are computationally efficient since small scale behaviors are not resolved but their integral effect on the large scale flow structure in the pool is taken into account.
\end{abstract}

\section{Introduction}

1.1. Motivation. A pressure suppression pool is an important part of a BWR reactor containment safety design. It serves as a heat sink and steam condenser to prevent containment pressure buildup during loss of coolant accident (LOCA) or during safety relief valve (SRV) opening in normal operations. Steam released from the reactor vessel is vented through the blowdown pipes (in case of LOCA) or through spargers (in case of SRV operation) and condenses in the pressure suppression water pool. The temperature of the pool gradually increases as a result of condensation. This leads to a reduction of the pool's pressure suppression capacity. Efficiency of the pool pressure suppression function is contingent upon the temperature of the pool surface, which determines the steam partial pressure in the wet well gas space. An increase of the pool's surface temperature due to stratification can lead to a significant increase in containment pressure [1]. Steam condensation stops when water at the level of the steam injection point and above reaches saturation temperature. The pool temperature is also important for emergency core cooling system (ECCS) which takes water from the pool and pumps it into the vessel to remove decay heat. ECCS pumps will be shut down to prevent cavitation if net positive suction head (NPSH) will drop below certain limit. The NPSH is also determined by water temperature in the pool. Cooling of the pool is implemented in order to keep water temperature low. However, cooling might be inefficient if stratification of the pool persists. Therefore the pool has to be mixed to increase pool's pressure suppression capacity, reduce containment pressure, and provide reliable ECCS operation. While pool mixing systems are also a part of the design, they are active nonsafety graded systems which can fail. The main motivation of this study is clarifying at which conditions steam injection itself can be regarded in safety analysis as a factor which can enhance pool mixing.

Steam injection in a pool of water is a source of both heat and momentum. A competition between heat 


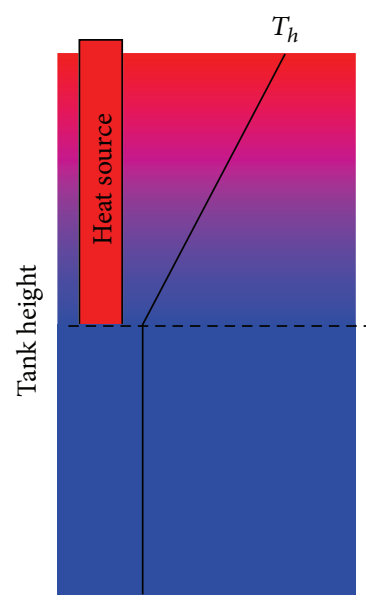

$T_{c}$

(a)

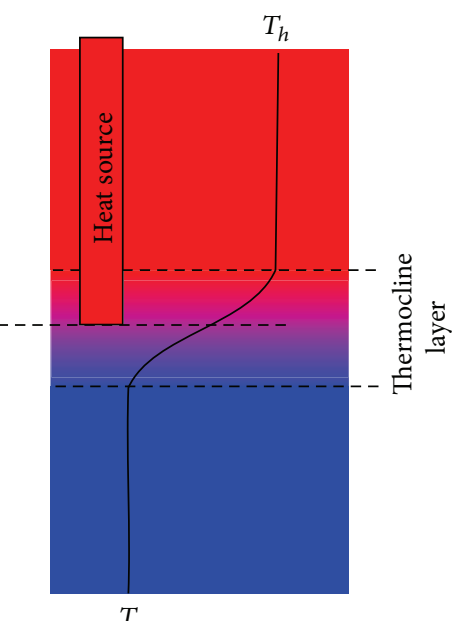

$T_{c}$

(b)
FIgURE 1: Typical configurations of thermal stratification in a tank (a) stratified layer and (b) thermocline layer. Note: $T_{h}$ : temperature of hot liquid; $T_{c}$ : temperature of cold liquid.

and momentum defines the pool state whether it is thermally mixed or stratified. The heat source induces the development of thermal stratification. The configuration of the stratified layers generally depends on the spatial distribution of the heat source and history of transient heat transfer in the pool (heating and cooling phases). In a BWR pressure suppression pool operation, thermal stratification development is caused by a heat source (such as a blowdown pipe or a sparger) immersed into the pool at a certain depth. There are two typical transient stratification configurations (as shown in Figure 1), (i) a stratified layer with continuous increase of water temperature from the bottom of the heat source but a constant temperature of cold water $T_{c}$ below the heat source (Figure 1(a)) and (ii) an isothermal top layer at temperature $T_{h}$ separated from the bottom layer of cold water by relatively thin thermocline layer where temperature is changing rapidly from $T_{c}$ to $T_{h}$ (Figure 1(b)). Such different configuration can exhibit different resistance to mixing.

The momentum induced by steam condensation is capable of creating large scale circulation which can mix the pool. However, mixing of a stratified pool takes some time which generally depends on the momentum rate. The time which is necessary to achieve mixing determines how fast the suppression pool's capacity can be restored. Thus, the characteristic mixing time scale is considered as an important parameter of the pool's operation.

1.2. State of the Art Review. The competition between the sources of heat and momentum is determined by the steam condensation regime. Condensation regimes of steam injection into a subcooled water pool at different conditions were studied intensively in the past [2-7]. Figure 2 depicts a 2D condensation regime map with dependencies on the steam mass flux and pool bulk temperature [2]. Recently, de With et al. [7] have compiled a 3D condensation regime diagram. The injector diameter was introduced as a third dimension

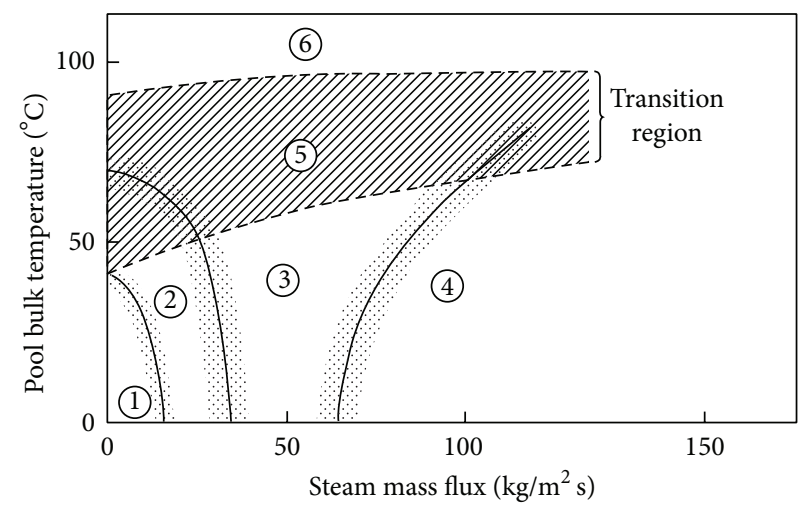

(1) Steam condensation within vents or blowdown pipes

(2) Chugging

(3) Condensation oscillations

(4) Quasi-steady condensation

(5) Transition region

(6) Incomplete condensation

FIGURE 2: Regime map of steam condensation [2].

in addition to the traditional water subcooling and steam mass fluxes. The 3D diagram is based on the data produced over three decades in different experiments with injector diameters up to $5 \mathrm{~cm}$.

A chugging regime $[2,8]$ can result in large amplitude oscillations of the steam-liquid interface. The momentum source created by such oscillations can enhance mixing [1]. Aya and Nariai $[9,10]$ studied chugging oscillations using a small scale experiment with a blowdown pipe of $18 \mathrm{~mm}$ in diameter and $1 \mathrm{~m}$ in height. The steam mass flow rate was in the range of $0.74 \sim 35 \mathrm{~g} / \mathrm{s}$. It was also shown that the analytical model proposed by Aya and Nariai is capable of reproducing the experimentally obtained frequency and amplitude of the oscillations.

Stratification and mixing phenomena in a large pool of water with a heat source have been studied experimentally and analytically. A strong stratification above a heat source submerged in a water pool was observed in different tests [11-20]. Heat transfer into the layer below the heat and momentum source was found to be limited by thermal conduction. The region below the source of heat remains inactive as a heat sink.

An experimental study of thermal stratification and mixing in relatively large pools was carried out in the PUMA facility [19]. It has been shown that the degree of thermal stratification in the suppression pool is strongly affected by the vent opening submergence depth, pool initial pressure, noncondensable gas flow rate, and steam injection rate and is less sensitive to the initial water temperature. Unfortunately, information provided in [19] is not sufficient to perform independent validation of codes and models against the PUMA data.

Similar experimental programs called POOLEX (POOL Experiment) and PPOOLEX (Pressurized POOLEX) [1114] have been performed at Lappeenranta University of Technology (LUT, Finland). The POOLEX facility is an open 
cylindrical stainless steel tank with an outer diameter of 2.4 $\mathrm{m}$ and a water pool depth of about $2.95 \mathrm{~m}$. Steam is injected through a submerged vertical blowdown pipe that has about $200 \mathrm{~mm}$ in inner diameter and is located close to the center of the tank. Heating and cooling phases were studied in the POOLEX tests.

It is instructive to note that flow regime domains observed in the POOLEX/PPOOLEX test agree rather well with the data from [20]. Apparent influence of chugging on mixing in the pool was observed in the POOLEX experiment [11]. The steam flow rate in the POOLEX STB-20 and STB-21 was kept below certain limit to prevent mixing in the pool by steam flow pulsations. In the STB-20 test, for example, the steam mass flow rate was kept in the range of $25-55 \mathrm{~g} / \mathrm{s}$ to make sure that steam condenses inside the blowdown pipe. In the STB-21 test, thermal stratification in the pool is formed with steam injection at small mass flow rate similar to the STB20 . Then the steam mass flow rate was increased up to $210 \mathrm{~g} / \mathrm{s}$ which resulted in an erosion of the thermal stratification layers and a uniform temperature (isothermal) distribution in the pool was observed. After a period of mixing, the steam mass flow rate was decreased at the level of $35 \mathrm{~g} / \mathrm{s}$ and thermal stratification started to develop again. In both experiments the duration of the heating phase was about 4 hours while the cooling phase took about 48 hours.

The POOLEX was later modified to become PPOOLEX (see Figure 3(a)), which has both a dry well $\left(\sim 13.3 \mathrm{~m}^{3}\right)$ and a wet well $\left(\sim 17.8 \mathrm{~m}^{3}\right)$. First, steam is injected through a horizontal inlet plenum, then injected into the dry well, and finally discharged into the wet well through a vertical blowdown pipe which is installed close to the central axis of the tank. A series of STR and MIX tests [12-14] have been performed in PPOOLEX to investigate thermal stratification and mixing. In the MIX-01 test (see Figure 3(b)), for example, there is a clearing phase which took about $500 \mathrm{~s}$ followed by development of thermal stratification for about 2200 s. An increase in steam mass flow rate resulted in thermal mixing that took about $300 \mathrm{~s}$ and remained thermally mixed until the end of the test. In the STR-02 test, as shown in Figure 3(c), a thermocline layer is observed in the water pool where the upper part is nearly isothermal with temperature increase up to $90^{\circ} \mathrm{C}$ while the temperature in the lower part remains uniform around $20^{\circ} \mathrm{C}$.

The availability of the detailed data from the POOLEX/PPOOLEX tests was instrumental for the development and validation of the approaches described in this work.

CFD modeling of POOLEX/PPOOLEX tests has been carried out by VTT, a technical research center in Finland. The direct contact condensation in short transients is directly simulated with different heat transfer correlations and interfacial surface area between the liquid and the vapor. The results showed that the condensation rate is very sensitive to the correlations. The oscillation frequencies of the steamwater interface in the blowdown pipe were much smaller than in the experiments [21-23].

Scaling approaches for prediction of thermal stratification and mixing in pools and in large interconnected enclosures were developed and applied by Peterson and coworkers at UC Berkeley [1, 24-29]. A 1D simulation code BMIX/BMIX++ was also developed to simulate stratification development in stably stratified conditions [27]. It was validated against a number of experiments [26-29]. However, BMIX++ does not predict a transient where initially stratified pool is gradually mixed, and thus it cannot help to infer about the time scale for such mixing processes.

Gamble et al. [1] studied postaccident long-term containment performance in case of passive SBWR containment and found that surface temperature of the pressure suppression pool is an important factor in determining the overall longterm containment pressure. Analytical models were developed and implemented into a 1D system simulation code, TRACG, and used to model thermal stratification behavior in a scaled test facility [1]. The main idea of the proposed model was based on analysis of the effect of injected momentum in each computational cell. Good agreement with the scaled experimental test data was reported. However such models are design specific and their validity in application to other designs and steam injection conditions is not clear.

Condensation and mixing phenomena during loss of coolant accident in a scaled down pressure suppression pool of simplified boiling water reactor were also studied by Norman et al. [20]. The results of their experiments were compared with the 1D thermal hydraulic code TRACE predictions and showed deficiency in the code capabilities to predict thermal stratification in the pool. Specifically, complete mixing was predicted using TRACE while thermal stratification was observed in the experiments.

An experimental investigation of steam condensation and CFD analysis of thermal stratification and mixing in subcooled water of the incontainment refueling water storage tank (IRWST) of advanced power reactor 1400 (APR1400) were performed by Song et al. [30], Kang and Song [31], and Moon et al. [32]. The IRWST is a BWR SP technology adopted in a PWR design to reduce the containment failure risk by condensing steam in a subcooled pool. A lumped volume condensation region model [31] was used to provide boundary conditions for temperature and velocity of the condensed steam and the entrained water in the CFD simulations. Their approach is similar to the model proposed earlier by Austin and Baisley [33]. However, these works address the case of steam injection through small holes (order of a centimeter in diameter) in a sparger as opposed to a blowdown pipe (about few hundred centimeters in diameter) that is considered in this paper. In addition, CFD codes, even with the condensation region model, are still too demanding for computational resources to be used for parameter sensitivity analysis in long transients (order of few days physical time).

The state of the art in understanding data and modeling capabilities relevant to suppression pool stratification and mixing phenomena can be summarized as follows.

(i) Numerous experimental studies were performed in the past on stratification and mixing in a pool, but only few are large scale tests with steam injection. Tests with steam injection have been carried 

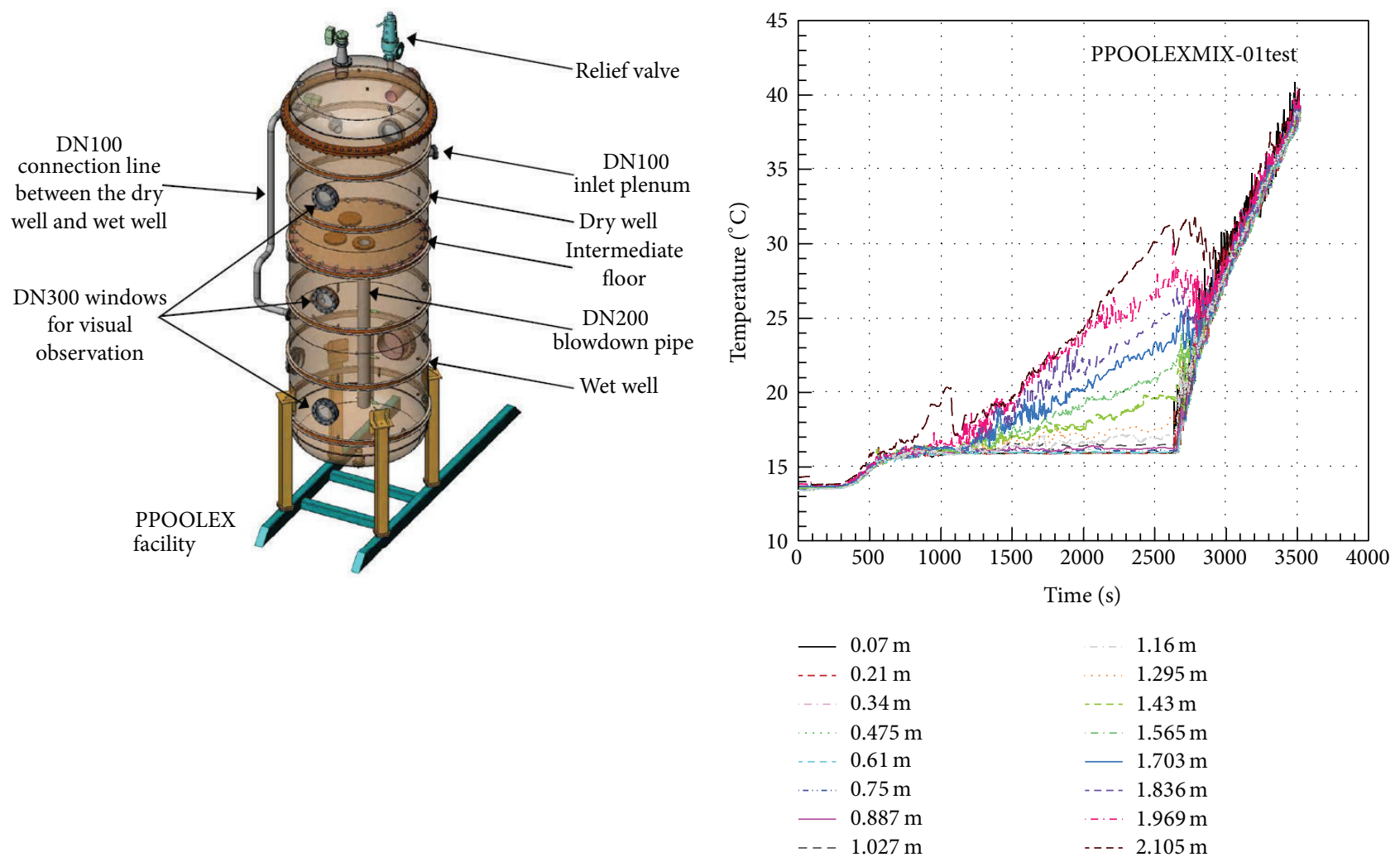

(a)

(b)
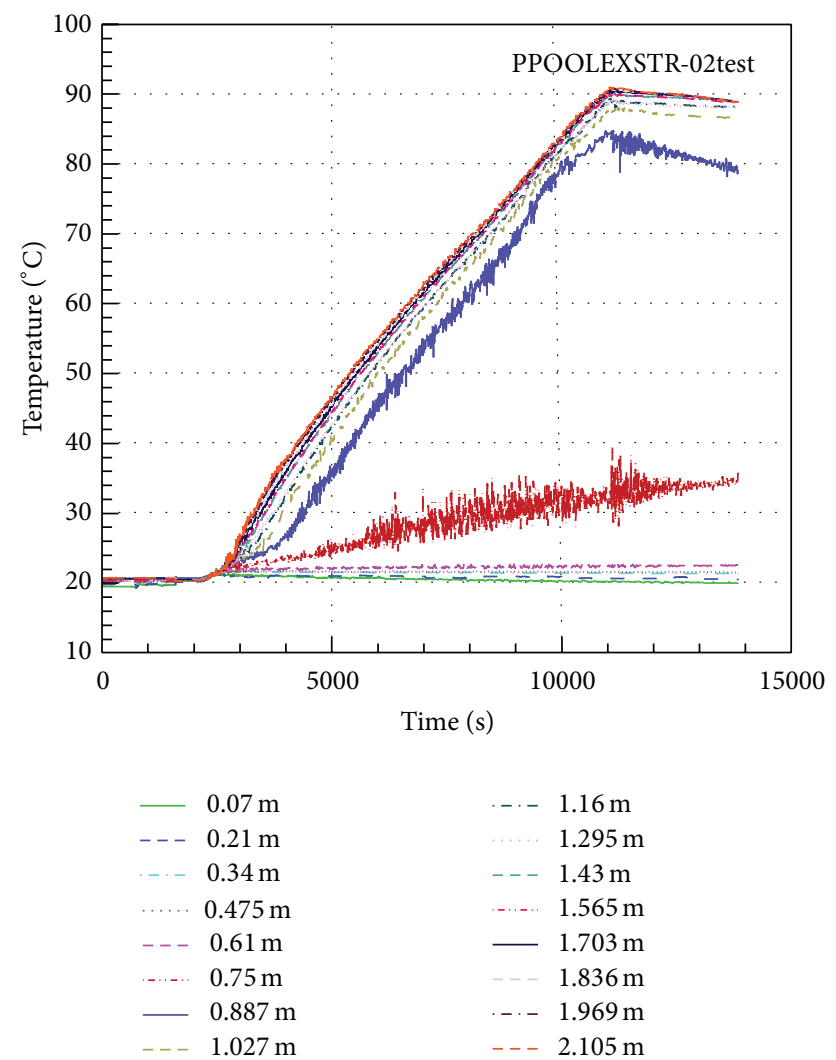

(c)

FIGURE 3: (a) PPOOLEX experiment facility and examples of (b) thermal stratification development during the MIX-01 test and (c) existence of a thermocline layer during the STR-02 test $[12,14]$. 
out mostly with small diameter pipes in order to clarify steam condensation regime and not in conjunction with mixing and stratification phenomena. Not all experimental data is readily available for model development and code validation. The POOLEX/PPOOLEX is a unique series of tests at relatively large scale which provides the most complete set of data on transient stratification and mixing caused by steam injection, which is necessary for code development and validation.

(ii) System thermal hydraulic 1D codes are unsuccessful in prediction of stratification development unless expressly developed and calibrated models and closures are provided. Lumped parameter and 1D models based on scaling approaches were developed and successfully used for modeling of thermal stratification development. Unfortunately, the applicability of these methods is limited to stably stratified or well mixed conditions. The time scale of transient stratified layer erosion has not been addressed with these models.

(iii) Direct application of fine resolution CFD (RANS, LES, and DNS) methods is not practical due to large uncertainty and excessive computing power in modeling of $3 \mathrm{D}$ high-Rayleigh-number natural convection flows in a large pool [34] and, most importantly, direct contact condensation on the steamwater interface [35].

1.3. Goal and Approach. The objective of the present work is to propose reliable and computationally efficient methods that can predict transient mixing and stratification phenomena induced by steam injection into a large pool. These methods are necessary for safety analysis of the pressure suppression pool's operations in different accident scenarios.

The main challenge of this work is how to take into account, in a robust and computationally efficient manner, the direct contact condensation (DCC) phenomena of steam injection into a subcooled pool that are important for development of stratification or mixing in the pool.

First, we stipulate that the goal of the analysis is to predict the stratification and mixing, and not DCC phenomena. Second, we recognize that the characteristic time and space scales of DCC phenomena are much smaller than the characteristic time and space scales of development of thermal stratification and global circulation and mixing in the pool. Third, we postulate that the individual details of small scale high frequency oscillations are lost due to the scale separation and only integral "net effects" of the DCC phenomena are important for mixing and stratification in a large pool. We describe these effects in terms of the heat and momentum sources induced by steam injection. In this work, we develop the "effective heat source" (EHS) model to provide the effect of steam injection on pool heat transfer as a distributed along pipe surface heat source and the "effective momentum source" (EMS) model to provide the effect of steam-water interface dynamic on the large scale circulation in the pool as a local source of momentum [36-39].
Thus, instead of "direct" CFD-type modeling of DCC phenomena, we propose to use the effective models (see also $[40,41])$ which can introduce the effect of DCC through appropriate boundary conditions implemented in $3 \mathrm{D}$ modeling of transient thermal stratification and mixing. It is instructive to note that such approach is close to the ideas proposed by Austin and Baisley [33] and later developed by Kang and Song [31] for horizontal steam injection at high velocities through relatively small nozzles of the spargers. In our work we address vertical injection of steam through large blowdown pipes at relatively low velocities. Also instead of CFD, we use as a computational vehicle in our analysis a thermal hydraulic code called GOTHIC, which was developed for containment analysis with the possibility to resolve $3 \mathrm{D}$ flow structures and has been extensively validated including stratification and mixing phenomena in large gas volumes [42-44]. GOTHIC provides a middleground approach between a lumped parameter approach and CFD. In each cell of a 3D grid, GOTHIC uses closures and correlations for simulation of heat, mass, and momentum transfer at subgrid scales using local cell parameters as an input. With such an approach the computational efficiency can be dramatically improved in comparison with standard CFD methods due to the much less strict demands for necessary grid resolution. For example, there is no need in GOTHIC to resolve near wall boundary layers, because heat and mass transfer is resolved by subgrid scale models based on boundary layer theories or experimental correlations. At the same time, 3D resolution of the flow field in GOTHIC is an advantage for the study of phenomena such as mixing and stratification, and it provides much greater flexibility than in OD and 1D models. A schematic of the EHS and EMS models is shown in Figure 4. One thermal conductor in GOTHIC is used to supply the equivalent heat flux through the pipe wall. One pump is used in GOTHIC to impose effective momentum into the water pool.

\section{Effective Heat Source (EHS) Model}

The EHS model provides integral heat source caused by steam injection. Its purpose is to conserve mass and thermal energy of injected steam. In Figure 4, the schematic of the EHS model is shown. It is assumed that only hot saturated water flows out of the blowdown pipe. This is the case also in reality when all steam condenses inside the blowdown pipe. Such approach correctly preserves the mass balance in the system even if some fraction of injected steam is condensed outside the pipe outlet.

A time-averaged mass flow $\left(\dot{\bar{M}}_{\text {steam }}\right)$ and enthalpy $\left(\bar{h}_{\text {steam }}\right)$ of the steam define the total effective heat source,

$$
\begin{aligned}
H_{\mathrm{eff}_{\text {total }}}(t) & =\dot{\bar{M}}_{\text {steam }}(t) \cdot \bar{h}_{\text {steam }}(t) \\
& =\frac{1}{\Delta t} \int_{t-\Delta t}^{t} \dot{M}_{\text {steam }}(\tau) \cdot h_{\text {steam }}(\tau) d \tau
\end{aligned}
$$




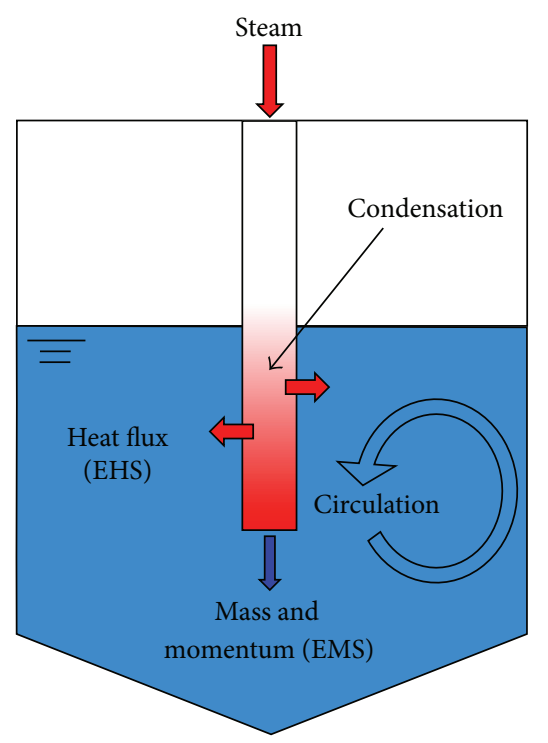

FIGURE 4: Schematic of effective heat source (EHS) and effective momentum source (EMS) models.

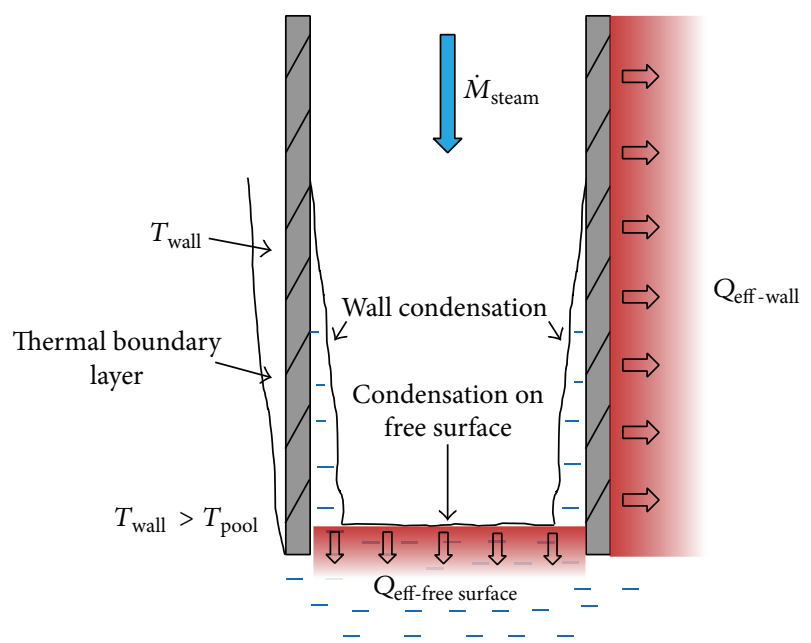

FIGURE 5: Condensation inside the blowdown pipe during steam injection.

while the spatial distribution of the effective heat source is determined as follows:

$$
\begin{gathered}
Q_{\mathrm{eff}_{\text {total }}}(t)=\int_{S} Q_{\mathrm{eff}}(\mathbf{x}, t) d S, \\
Q_{\mathrm{eff}}(\mathbf{x}, t)=\frac{1}{\Delta t} \int_{t-\Delta t}^{t} \mathrm{Q}(\mathbf{x}, \tau) d \tau,
\end{gathered}
$$

where $Q_{\text {eff }}(\mathbf{x}, t)$ denotes the spatial $(x)$ distribution (flux) of effective (time-averaged) heat source at time moment $t$; $\Delta t$ is the time-averaging interval which is considerably larger than the characteristic time scale of DCC oscillations; $Q(\mathbf{x}, \tau)$ is the local instantaneous heat flux through $S$ which denotes the surface of the pipe wall and pipe outlet.

The schematic illustration of the steam condensation inside the blowdown pipe is shown in Figure 5. Steam directed through the blowdown pipe can condense on the walls and on the free water surface which results in local heat fluxes on the walls and on the free surface close to the outlet. The spatial distribution of the effective heat source depends on the steam flow rate and condensation regime. For example, if the steam mass flow rate and temperature of the surrounding pool are relatively low, the condensation and thus effective heat source can be more or less uniformly distributed along the submerged surface of the blowdown pipe. With significantly higher steam mass flow rates or closer to saturation pool temperature in the upper layer, steam condensation inside the blowdown pipe is limited and most of condensation will occur in the vicinity of the blowdown pipe outlet. While detailed modeling of such phenomena is possible in the GOTHIC code, it is beyond the scope of current work. In order to demonstrate the feasibility of the proposed approach we consider two limiting cases in the EHS model with respect to the distribution of a total heat flux $Q_{\text {eff }_{\text {total }}}$ : (i) the total effective heat rate is distributed uniformly along the pipe walls $Q_{\text {eff-wall }}=Q_{\text {eff }_{\text {total }}}$; or (ii) the total effective heat rate is applied at the pipe outlet free surface, $Q_{\text {eff-free surface }}=Q_{\text {eff }_{\text {total }}}$. In reality, intermediate states are possible with arbitrary redistribution of the total heat flux between the pipe wall and outlet. However, as we will show later, one of the limiting cases provides sufficiently accurate results for the POOLEX/PPOOLEX experiments where typical steam flow rates during the thermal stratification phase are between 50 and $60 \mathrm{~g} / \mathrm{s}$, with respective heat rate between 112 and $134 \mathrm{~kW}$, while steam flow rates during the mixing phase are between 200 and $425 \mathrm{~g} / \mathrm{s}$, with respective heat rate between 446 and $948 \mathrm{~kW}$.

\section{Effective Momentum Source (EMS) Model}

The EMS model provides time-averaged momentum source induced by steam injection. This momentum creates large scale circulation in the pool which can lead to erosion of thermally stratified layer and mixing of the pool. The effective momentum source is calculated by

$$
\mathbf{M}_{\mathrm{eff}}(t)=\frac{1}{\Delta t} \int_{t-\Delta t}^{t} \mathbf{M}(\tau) d \tau .
$$

It is instructive to note that steam condensation can induce different time-averaged (effective) momentum rates at the same mass flow rate of steam depending on the condensation regime. Thus the goal of the EMS model is to establish a connection between steam injection parameters, pool conditions, and resulting momentum injected into the pool.

For a given diameter of the blowdown pipe the condensation regime depends on the injected steam flux and pool bulk temperature (see Figure 2). In the POOLEX/PPOOLEX experiments, the condensation regime typically starts from condensation within the pipe (region 1) and then goes to chugging (region 2) as the steam flow rates are increased and in some tests it goes further to the transition regime (region 5) as the pool bulk temperature increases. According to the observed pool mixing rate in PPOOLEX tests $[13,14]$, the effective momentum source during condensation 
within the pipe regime is negligible compared to the effective momentum during chugging. The effective momentum in the chugging regime is also higher than that in the transition regime, although the steam mass flow rate can be higher in the transition regime.

The reason is that steam injected into a subcooled pool creates different patterns of fluid oscillations in different regimes. For instance, in the chugging regime, large amplitude periodic oscillations of the free surface inside the pipe are caused by the periodic process of (i) steam injection, (ii) expansion of the steam bubble around the pipe outlet, and (iii) volumetric condensation inside of the overexpanded steam followed by (iv) sudden bubble collapse and suction of water inside the blowdown pipe. No steam bubble plume is injected into the pool above the pipe outlet. As shown in Figure 6, the fluid motion inside the pipe during each cycle of the chugging can be separated into two parts: water injection in downward direction and then suction of water in upward direction during steam condensation phase. Both phases of such quasiperiodic motion introduce a momentum source that affects large scale circulation flow in the pool.

In this work we consider a specific case when the momentum is generated mostly by the oscillatory flow in the blowdown pipe. The other case, when steam and possibly noncondensable gases escaping the pipe can contribute to generation of momentum in the pool due to the buoyancy force, is beyond the scope of this work.

3.1. Synthetic Jet Model for Prediction of the Effective Momentum Source. The "synthetic jet" term was introduced to denote fluid motion which can be generated by oscillatory flow through an orifice with zero time-averaged mass flow [45]. The vibrations of a diaphragm inside an enclosed volume with single orifice are usually used in order to create a synthetic jet (Figure $7(\mathrm{a})$ ). It has been shown that the injection phase of the oscillatory flow creates a train of vortices which has enough thrust to propagate and is not destroyed during the suction phase (see Figure $7(\mathrm{~b})$ ). The resulting (synthetic) jet is responsible for the far-field quasi-steady flow. In an axisymmetric case, a criterion for jet formation $[46,47]$ is given as $L /(2 \pi d)>0.16$, where $L$ is called the stroke length and $d$ is the diameter of the orifice. Early experiments by Smith and Glezer [48] have shown that a low Reynolds number synthetic jet has many characteristics that resemble continuous higher Reynolds number jets. Mallinson et al. [49] have also shown that the far-field behavior of round synthetic jets is closer to that of conventional (turbulent) round jets; that is, the centerline velocity decays like $1 / x$.

For a single harmonic oscillation, the velocity scale based on the momentum flux [50] is given as

$$
U_{0}=\sqrt{2} f L,
$$

where $f$ is the frequency of oscillation and $L$ is the amplitude of oscillation. The momentum rate is then given as

$$
M=\frac{\pi \rho U_{0}^{2} d^{2}}{4},
$$

where $\rho$ is the liquid density and $d$ is the diameter of blowdown pipe.

In this work we use similarities between basic physics of synthetic jets and flow created by the free surface oscillations in the blowdown pipe in order to propose a model for effective momentum. Indeed, in case of condensation oscillations, the velocity of periodic oscillations is usually much larger than the velocity determined by the mass flow of steam, while in the synthetic jet case, the mass flow through the orifice is exactly zero. Similar to the synthetic jet, the large scale circulation in the pool does not follow the high frequency oscillations of the water level in the pipe; that is, the flow pattern in the pool is not oscillatory.

Our hypothesis, which is validated in [51], is that the effective momentum source in case of steam injection into subcooled water can be predicted using the synthetic jet model that is based on (4) and (5). Such characteristics as frequency and stroke lengths (or amplitude) of the oscillations should be known in order to do that.

The amplitude and frequency of the water level oscillations in the pipe can be obtained experimentally, for example, by temperature measurements on the pipe's inner surface or by a level meter. Figure 8(a) shows a sample 5-second time window of TC measurements inside the blowdown pipe during chugging in PPOOLEX MIX-01 test. The corresponding water level positions inside the pipe are shown in Figure 8(b). To calculate the effective momentum given the TC measurements inside the blowdown pipe, the following steps are implemented.

(1) Convert the TC measurements to water level positions (see Figure 8 as an example).

(2) Calculate the velocities $u=d z / d t$, where $z$ is the water level position.

(3) Calculate the moving time-averaged velocities by

$$
\bar{U}(t)=\sqrt{\frac{1}{\Delta t} \int_{t-\Delta t}^{t} u^{2}(\tau) d \tau}
$$

with an averaging time scale $\Delta t=100 \mathrm{~s}$.

(4) Calculate the effective (jet) velocity $U_{0}(t)=(2 / \pi)$. $\bar{U}(t)$. This relation can be shown simply by taking a single harmonic signal $z(t)=L \cdot \sin (2 \pi f t)$ where $f=$ $1 / T$ and use (4) and (6).

(5) Calculate the effective momentum rate $M_{\text {eff }}$ given by (5).

For the $5 \mathrm{~s}$ time window given in Figure 8(b), the timeaveraged velocities are around $0.65 \mathrm{~m} / \mathrm{s}$ and the momentum rates are around $14.6 \mathrm{~kg} \cdot \mathrm{m} / \mathrm{s}^{2}$.

The ultimate goal of the EMS model is to calculate the effective momentum $M_{\text {eff }}$ (see Figure 9), given the steam mass flux, pool bulk temperature, and design specific parameters. The first step is to determine the condensation regime. The $2 \mathrm{D}$ condensation regime map of Lahey and Moody [2] is found to be sufficient for large scale diameters of the pipe $(\sim 200 \mathrm{~mm})$. Next, given the condensation regime and design specific 


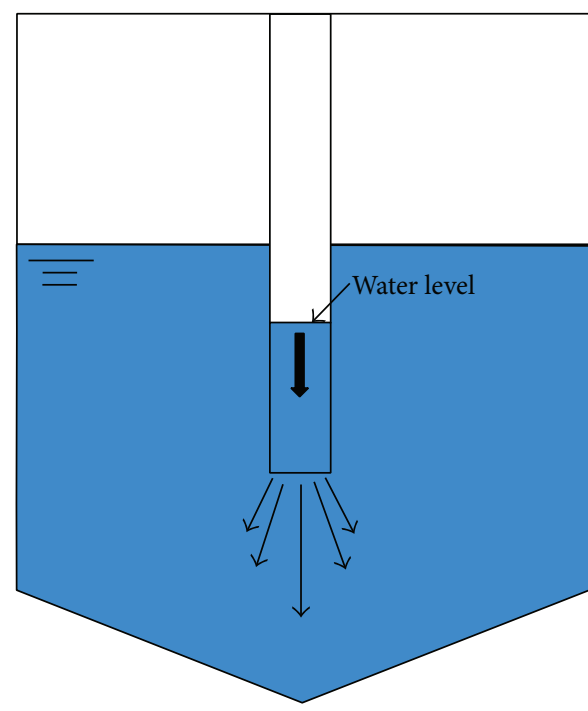

(a)

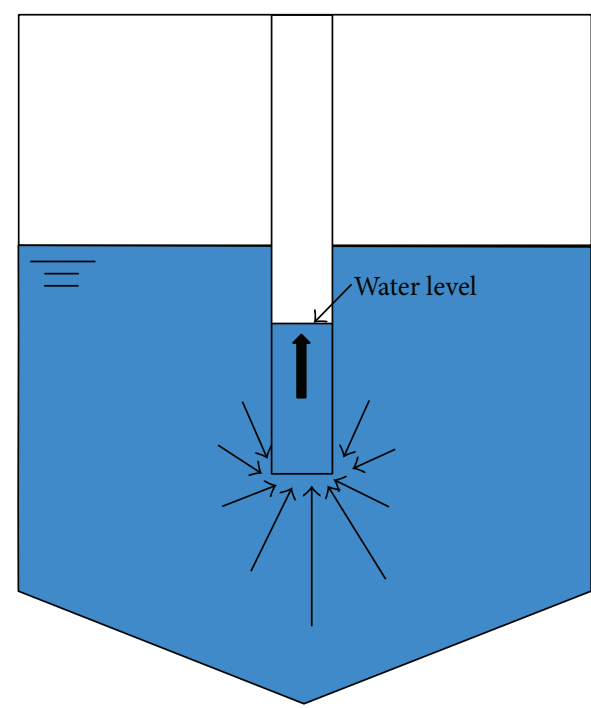

(b)

FIGURE 6: Separate effect during chugging regime when steam is injected through vertical pipe, (a) injection phase and (b) suction phase.

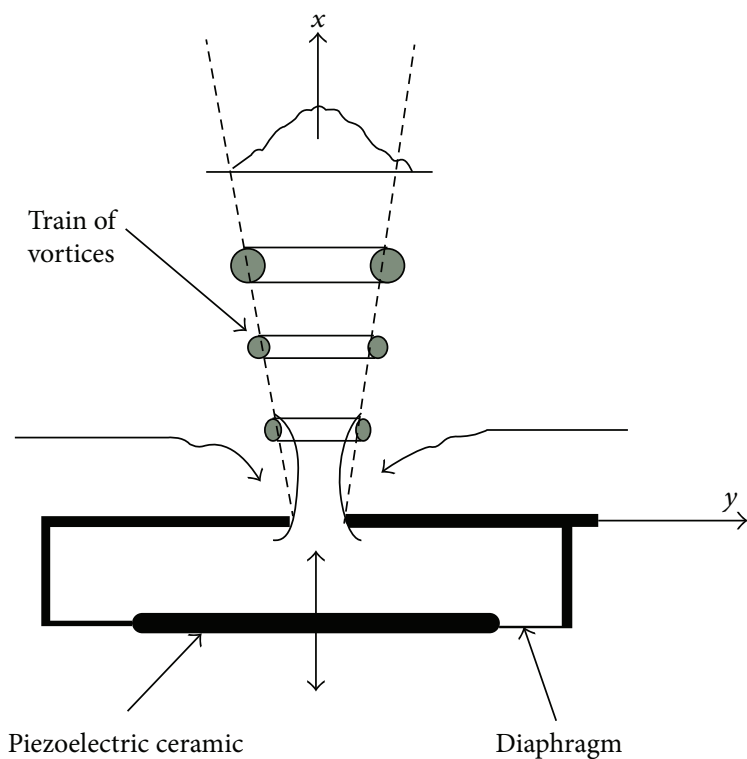

(a)

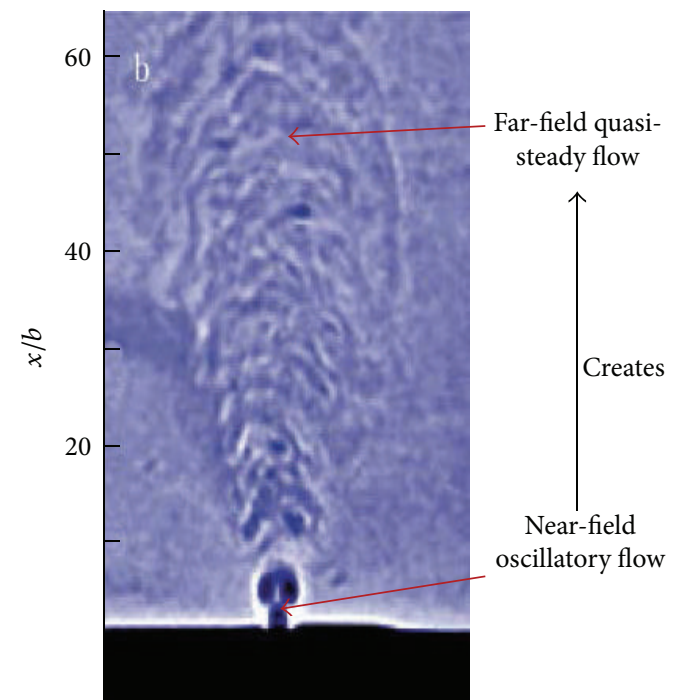

(b)

FIGURE 7: (a) Schematic of a synthetic jet actuator and (b) Schlieren image of a rectangular synthetic jet [48].

parameters, the frequency and amplitude of oscillations in the pipe (if they occur) should be obtained from the experiment or predicted using such models as the Aya and Nariai model [52]. However, scalability of the Aya and Nariai model for large injection diameters is still an issue and is currently being investigated. The last step is the calculation of the effective momentum that is based on synthetic jet theory. In the synthetic jet theory, the amplitude and frequency of oscillations inside the pipe are directly proportional to the velocity scale and hence the effective momentum.

\section{Summary}

In order to enable sufficiently accurate and computationally affordable simulations of thermal stratification and mixing during a steam injection into a large pool of water, the concepts of effective heat source (EHS) and effective momentum source (EMS) models are proposed in this work. Specifically, the EHS/EMS models are developed for steam injection through a single vertical pipe submerged in a pool under two condensation regimes: complete condensation inside 


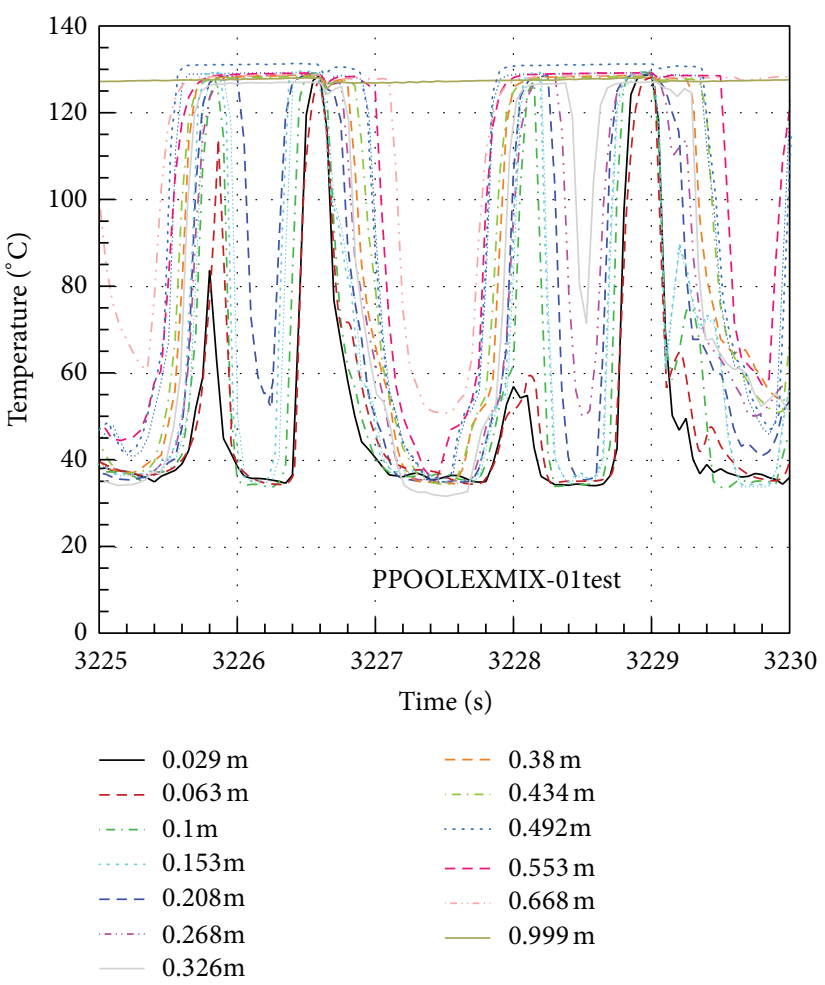

(a)

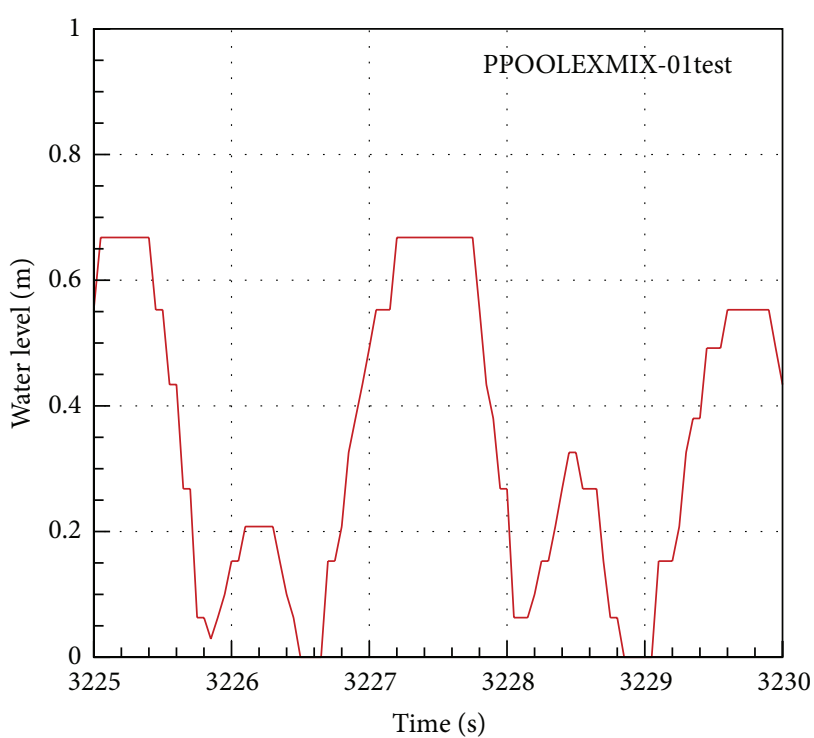

(b)

FIGURE 8: (a) TC measurements inside the blowdown pipe and corresponding (b) water level positions for a $5 \mathrm{~s}$ time window and superimposed smoothed data with a moving average filter. Frequency and amplitude of oscillations can be based on the water level positions inside the pipe. The outlet of the pipe is at $0 \mathrm{~m}$.

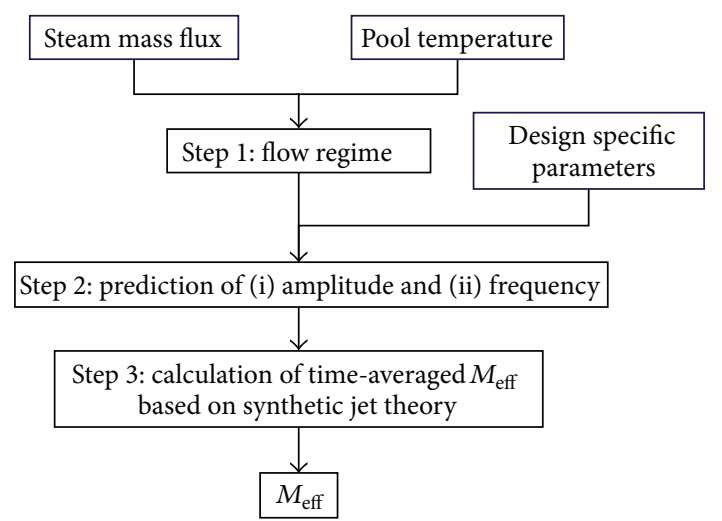

FIGURE 9: Calculation diagram for the effective momentum $M_{\text {eff }}$ with input of steam mass flux, pool temperature, and design specific parameters.

the pipe and chugging. These models are computationally efficient since small time and space scale behaviors are not resolved directly but their integral effect on the large scale flow structure in the pool is taken into account. The EMS model is based on the synthetic jet model which has to be complemented with the data about amplitude and frequency of the condensation oscillations in different flow regimes. In [51], the EHS/EMS models are implemented in GOTHIC and then validated against a number of thermal stratification and mixing tests carried out in POOLEX/PPOOLEX facility.

\section{Conflict of Interests}

The authors declare that there is no conflict of interests regarding the publication of this paper.

\section{Acknowledgments}

Support from the NORTHNET RM3 and Nordic Nuclear Safety Research (NKS) is greatly acknowledged. The authors are grateful to Markku Puustinen (LUT) and coworkers for very fruitful discussions and providing unique experimental data for the development and validation of the models.

\section{References}

[1] R. E. Gamble, T. T. Nguyen, B. S. Shiralkar, P. F. Peterson, R. Greif, and H. Tabata, "Pressure suppression pool mixing in passive advanced BWR plants," Nuclear Engineering and Design, vol. 204, no. 1-3, pp. 321-336, 2001.

[2] R. T. Lahey and F. J. Moody, The Thermal Hydraulics of a Boiling Water Reactor, American Nuclear Society, La Grange Park, Ill, USA, 2nd edition, 1993. 
[3] C. K. Chan and C. K. B. Lee, "A regime map for direct contact condensation," International Journal of Multiphase Flow, vol. 8, no. 1, pp. 11-20, 1982.

[4] K. Liang and P. Griffith, "Experimental and analytical study of direct contact condensation of steam in water," Nuclear Engineering and Design, vol. 147, no. 3, pp. 425-435, 1994.

[5] S. Cho, C. H. Song, C. K. Park, S. K. Yang, and M. K. Chung, "Experimental study on dynamic pressure pulse in direct contact condensation of steam jets discharging into subcooled water," in Proceedings of the Japan-Korea Symposium on Nuclear Thermal Hydraulics and Safety (NTHAS '98), vol. 291, 1997.

[6] D. H. Youn, K. B. Ko, Y. Y. Lee, M. H. Kim, Y. Y. Bae, and J. K. Park, "The direct contact condensation of steam in a pool at low mass flux," Journal of Nuclear Science and Technology, vol. 40, no. 10, pp. 881-885, 2003.

[7] A. P. de With, R. K. Calay, and G. de With, "Three-dimensional condensation regime diagram for direct contact condensation of steam injected into water," International Journal of Heat and Mass Transfer, vol. 50, no. 9-10, pp. 1762-1770, 2007.

[8] G. W. Fitzsimmons, D. L. Galyard, R. B. Nixon, M. J. Mann, and K. P. Yu, "Mark I Containment Program, Full Scale Test Program Final Report,” General Electric Report NEDE-24539, 1979.

[9] I. Aya and H. Nariai, "Chugging phenomenon induced by steam condensation into pool water (amplitude and frequency of fluid oscillation)," Heat Transfer-Japanese Research, vol. 14, no. 4, pp. 26-43, 1985.

[10] I. Aya, H. Nariai, and M. Kobayashi, "Pressure and fluid oscillations in vent system due to steam condensation (I), experimental results and analysis model for chugging," Journal of Nuclear Science and Technology, vol. 17, no. 7, pp. 499-515, 1980.

[11] J. Laine and M. Puustinen, “Thermal stratification experiments with the condensation pool test rig," NKS-117, 2006.

[12] M. Puustinen, J. Laine, and A. Räsänen, "PPOOLEX experiments on thermal stratification and mixing," Research Report CONDEX 1/2008, NKS-198, 2009.

[13] J. Laine, M. Puustinen, A. Räsänen, and V. Tanskanen, "PPOOLEX experiments on stratification and mixing in the wet well pool," NKS-240, 2011.

[14] J. Laine, M. Puustinen, and A. Räsänen, "PPOOLEX experiments on dynamics of free water surface in the blowdown pipe," Research Report EXCOP 2/2012, NKS-281, 2013.

[15] P. F. Peterson, I. J. Rao, and V. E. Schrock, "Transient thermal stratification in pools with shallow buoyant jets," in Nuclear Reactor Thermal Hydraulics, HTD, Y.A. Hassan and L.E. Hochreiter, Eds., vol. 190, pp. 55-62, ASME, New York, NY, USA, 1991.

[16] Y. Kataoka, T. Fukui, and S. Hatamiya, "Experimental study on convection heat transfer along a vertical flat plate between different temperature pools," in Proceedings of the ANS National Heat Transfer Conference, pp. 28-31, Minneapolis, Minn, USA, July 1991.

[17] R. J. Fox, "Temperature distribution in pools with shallow buoyant jets," in Proceedings of the 5th International Topical Meeting on Nuclear Reactor Thermal Hydraulics (NURETH-5 '92), pp. 1227-1234, Salt Lake City, Utah, USA, September 1992.

[18] B. L. Smith, T. V. Dury, M. Huggenberger, and N. Nöthiger, "Analysis of single-phase mixing experiments in open pools," in Thermal Hydraulics of Advanced and Special Purpose Reactors, ASME HTD, F. B. Cheung and P. F. Peterson, Eds., vol. 209, pp. 91-100, ASME, New York, NY, USA, 1992.
[19] L. Cheng, K. S. Woo, M. Ishii, J. Lim, and J. Han, "Suppression pool mixing and condensation tests in PUMA facility," in Proceedings of the International Conference on Nuclear Engineering (ICONE '06), 2006.

[20] T. L. Norman, H. S. Park, S. T. Revankar, M. Ishii, and J. M. Kelly, "Thermal stratification and mixing in an open water pool by submerged mixtures of steam and air," in Proceedings of the ASME International Mechanical Engineering Congress and Exposition (IMECE '06), November 2006.

[21] A. Timperi, T. Pättikangas, and J. Niemi, "Analysis of Loads and Fluid-Structure Interactions in a Condensation Pool," Research Report NKS-154, Nordic Nuclear Safety Research, 2007.

[22] T. Pättikangas, J. Niemi, and A. Timperi, "CFD and FEM modeling of PPOOLEX experiments," Research Report NKS236, Nordic Nuclear Safety Research, 2011.

[23] T. Pättikangas, J. Niemi, and A. Timperi, "Numerical modelling of pressure suppression pools with CFD and FEM codes," Research Report NKS-249, Nordic Nuclear Safety Research, 2011.

[24] P. F. Peterson, "Scaling and analysis of mixing in large stratified volumes," International Journal of Heat and Mass Transfer, vol. 37, no. 1, pp. 97-106, 1994.

[25] P. F. Peterson and R. Gamble, "Scaling for forced-convection augmentation of heat and mass transfer in large enclosures by injected jets," Transactions of the American Nuclear Society, vol. 78, pp. 265-266, 1998.

[26] S. Kuhn, H. K. Kang, and P. F. Peterson, "Study of mixing and augmentation of natural convection heat transfer by a forced jet in a large enclosure," Journal of Heat Transfer, vol. 124, no. 4, pp. 660-666, 2002.

[27] H. Zhao, Computation of mixing in large stably stratified enclosures [Ph.D. thesis], University of California, Berkeley, Calif, USA, 2003.

[28] F. Niu, H. Zhao, P. F. Peterson, J. Woodcock, and R. E. Henry, "Investigation of mixed convection in a large rectangular enclosure," Nuclear Engineering and Design, vol. 237, no. 10, pp. 1025-1032, 2007.

[29] H. Zhao and P. F. Peterson, "One-dimensional analysis of thermal stratification in AHTR and SFR coolant pools," in Proceeding of the 12th International Topical Meeting on Nuclear Reactor Thermal Hydraulics (NURETH-12 '07), Pittsburgh, Pa, USA, October 2007.

[30] C. H. Song, W. P. Baek, M. K. Chung, and J. K. Park, "Multidimensional thermal-hydraulic phenomena in advanced nuclear reactor systems: current status and perspectives of the R\&D program at KAERI," in Proceedings of the International Conference on Nuclear Reactor Thermal Hydraulics (NURETH '03), Paper I00121, Seoul, Republic of Korea, October 2003.

[31] H. S. Kang and C. H. Song, "CFD analysis for thermal mixing in a subcooled water tank under a high steam mass flux discharge condition," Nuclear Engineering and Design, vol. 238, no. 3, pp. 492-501, 2008.

[32] Y. Moon, H. Lee, and G. Park, "CFD simulation of steam jet-induced thermal mixing in subcooled water pool," Nuclear Engineering and Design, vol. 239, no. 12, pp. 2849-2863, 2009.

[33] S. Austin and D. Baisley, "System $80+$ summary of program to evaluate DCRT issues related to the safety depressurization system and IRWST-task 12," ABB-CE Documentation, 1992.

[34] R. R. Nourgaliev and T. N. Dinh, "The investigation of turbulence characteristics in an internally-heated unstably-stratified fluid layer," Nuclear Engineering and Design, vol. 178, no. 3, pp. 235-258, 1997. 
[35] V. Tanskanen, D. Lakehal, and M. Puustinen, "Validation of direct contact condensation CFD models against condensation pool experiment," in Proceedings of Experiments and CFD Code Applications to Nuclear Reactor Safety (XCFD4NRS OECD '08), Grenoble, France, September 2008.

[36] H. Li and P. Kudinov, "GOTHIC code simulation of thermal stratification in POOLEX facility," Research Report NKS-196, 2009.

[37] H. Li, P. Kudinov, and W. Villanueva, "Modeling of condensation, stratification, and mixing phenomena in a pool of water," Nordic Nuclear Safety Research, Research Report NKS-225, 2010.

[38] H. Li, P. Kudinov, and W. Villanueva, "Development and validation of effective models for simulation of stratification and mixing phenomena in a pool of water," Research Report NKS248, Nordic Nuclear Safety Research, Roskilde, Denmark, 2011.

[39] H. Li, W. Villanueva, and P. Kudinov, "Effective momentum and heat flux models for simulation of stratification and mixing in a large pool of water," Nordic Nuclear Safety Research, Research Report NKS-266, 2012.

[40] H. Li and P. Kudinov, "An approach toward simulation and analysis of thermal stratification and mixing in a pressure suppression pool," Paper 243, NUTHOS-7, Seoul, Republic of Korea, 2008.

[41] H. Li and P. Kudinov, "An approach for simulation of mixing in a stratified pool with the GOTHIC code," ANS Transactions, 2009.

[42] "GOTHIC containment analysis package qualification report," Version 8.0 (beta 1), NAI 8907-09 Rev 12, 2010.

[43] M. Andreani, "Pretest calculations of phase A of ISP-42 (PANDA) using the gothic containment code and comparison with the experimental results," Nuclear Technology, vol. 148, no. 1, pp. 35-47, 2004.

[44] M. Andreani and F. Putz, "Simulation of gas mixing and inter-compartment transport using the GOTHIC code," in Proceedings of the 11th International Conference on Nuclear Engineering (ICONE '03), Tokyo, Japan, April 2003.

[45] B. L. Smith and G. W. Swift, "A comparison between synthetic jets and continuous jets," Experiments in Fluids, vol. 34, no. 4, pp. 467-472, 2003.

[46] Y. Utturkar, R. Holman, R. Mittal, R. Carroll, B. Sheplak, and L. Cattafesta, "A jet formation criterion for synthetic jet actuators," in Proceedings of the 41st Aerospace Sciences Meeting and Exhibit, AIAA Paper 2003-0636, Reno, Nev, USA, 2003.

[47] Q. Gallas, R. Holman, T. Nishida, B. Carroll, M. Sheplak, and L. Cattafesta, "Lumped element modeling of piezoelectric-driven synthetic jet actuators," AIAA Journal, vol. 41, no. 2, pp. 240-247, 2003.

[48] B. L. Smith and A. Glezer, "The formation and evolution of synthetic jets," Physics of Fluids, vol. 10, no. 9, pp. 2281-2297, 1998.

[49] S. Mallinson, G. Hong, and J. Reizes, "Some characteristics of synthetic Jets," in Proceedings of the 30th AIAA Fluid Dynamics Conference, AIAA Paper 1999-3651, Norfolk, Va, USA, 1999.

[50] B. Smith and G. Swift, "Synthetic jets at larger reynolds number and comparison with continuous jets," in Proceedings of the 31st AIAA Fluid Dynamics Conference and Exhibit, AIAA Paper 2001-3030, Anaheim, Calif, USA, 2001.

[51] H. Li, W. Villanueva, and P. Kudinov, "Validation of effective momentum and heat flux models for stratification and mixing in a water pool," Research Report NKS-284, Nordic Nuclear Safety Research, Roskilde, Denmark, 2013.
[52] H. Nariai and I. Aya, "Fluid and pressure oscillations occuring at direct contact condensation of steam flow with cold water," Nuclear Engineering and Design, vol. 95, pp. 35-45, 1986. 


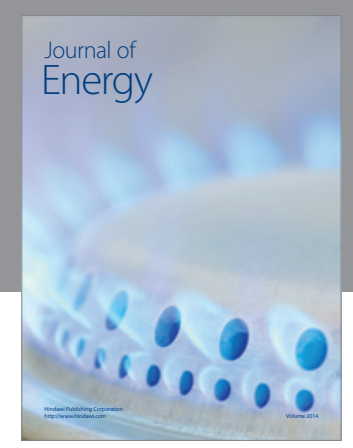

Journal of

Industrial Engineering
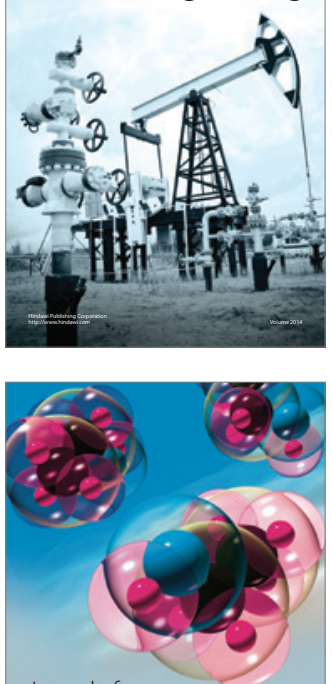

Fuels
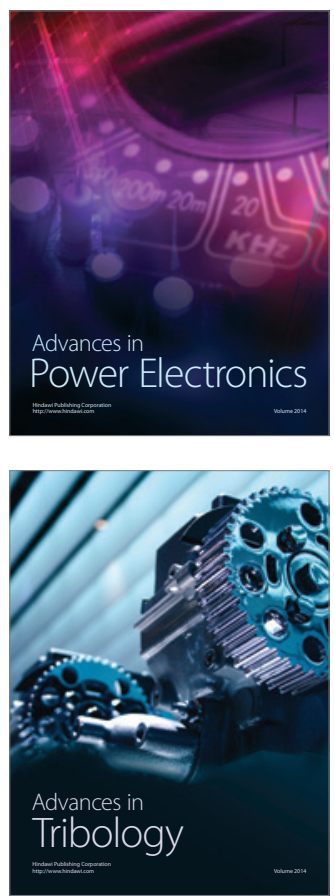

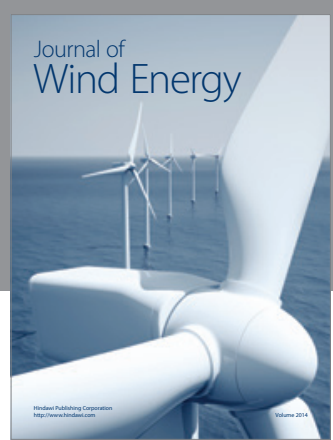

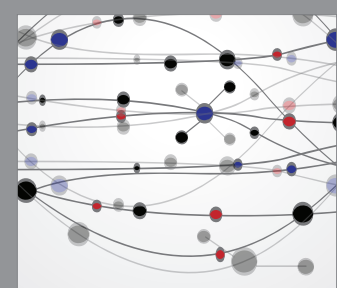

The Scientific World Journal

Submit your manuscripts at http://www.hindawi.com

Journal of

Structures
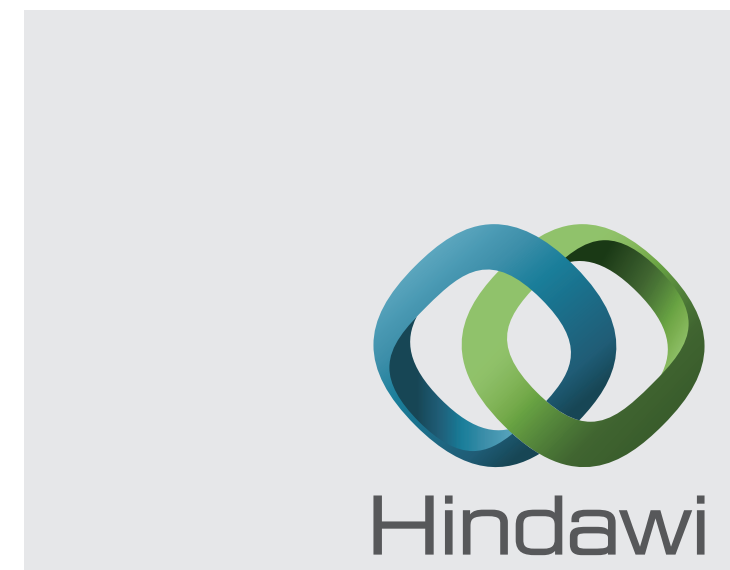

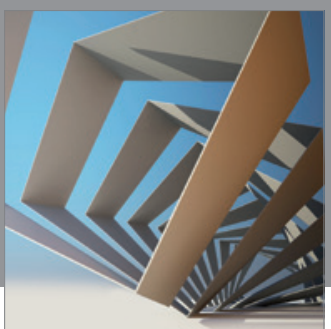

Rotating

Machinery
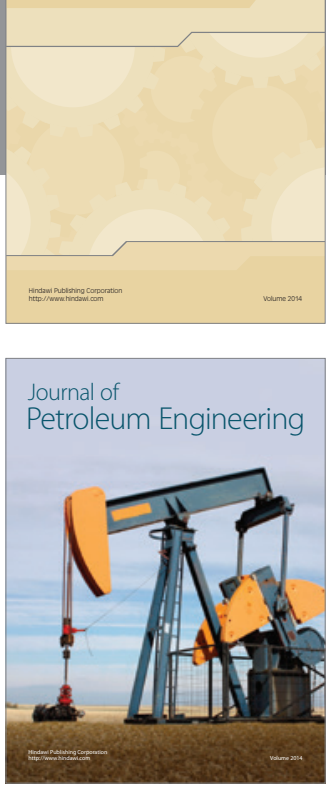

Journal of

Solar Energy
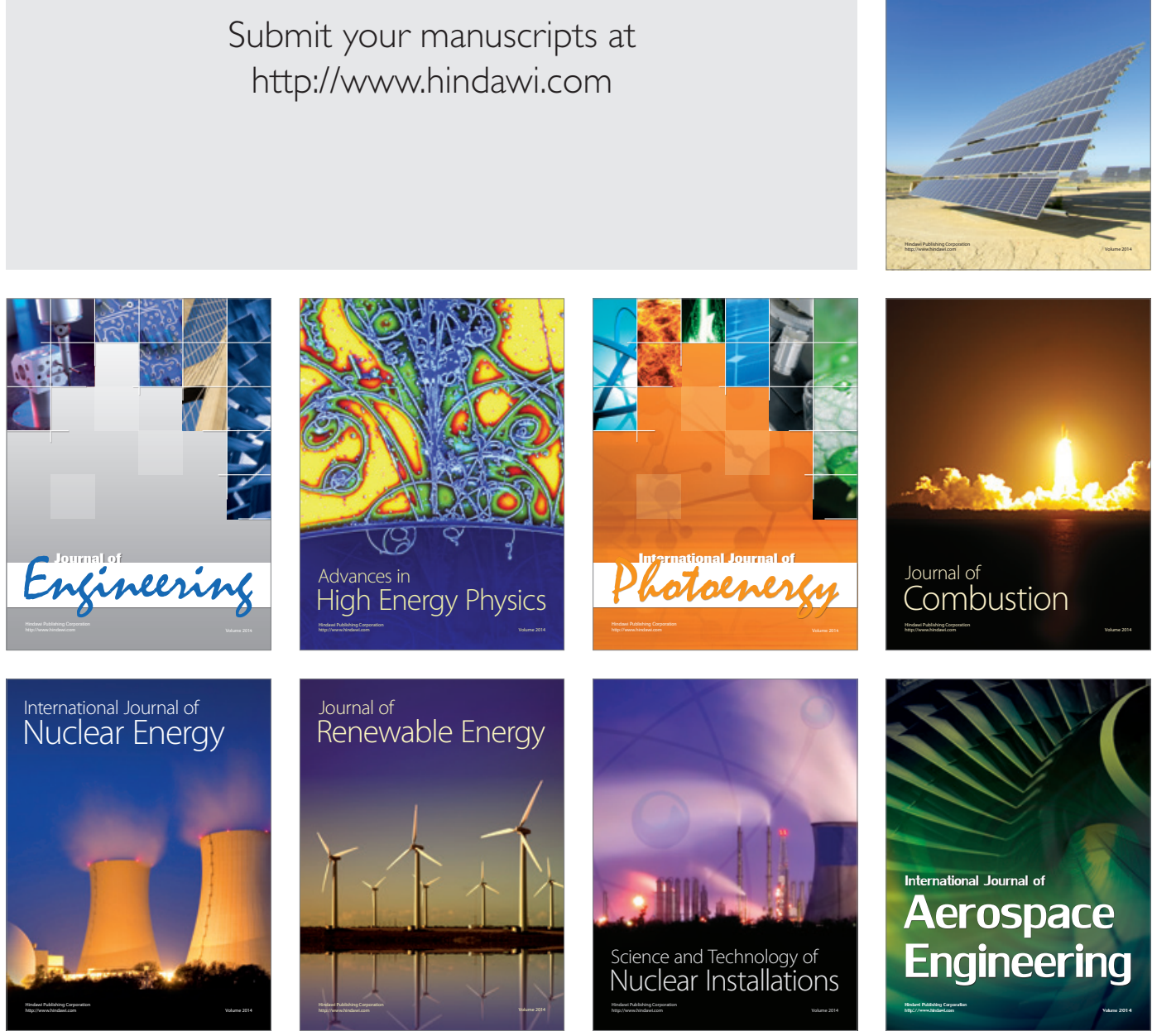